\title{
Modeling in-sewer transformations at catchment scale - implications on drug consumption estimates in wastewater-based epidemiology
}

Ann-Kathrin McCall a , Rocco Palmitessa ${ }^{\text {b }}$, Frank Blumensaat ${ }^{\mathrm{a}, \mathrm{c}}$, Eberhard Morgenroth ${ }^{\mathrm{a}, \mathrm{c}}$, Christoph

$$
\operatorname{Ort}^{\mathrm{a}, *}
$$

a Eawag, Swiss Federal Institute of Aquatic Science and Technology, CH 8600 Dübendorf, Switzerland

${ }^{b}$ University of Padova, 35131 Padova, Italy

${ }^{c}$ ETH Zürich, Institute of Environmental Engineering, 8093 Zürich, Switzerland

* Corresponding author. Tel.: +41 58765 5277. E-mail address: Christoph.Ort@eawag.ch

This accepted manuscript is made available under the CC-BY-NC-ND 4.0 license http:// creativecommons.org/licences/by-nc-nd/4.0/ 


\begin{abstract}
To which extent illicit drugs are transformed during in-sewer transport, depends on a number of factors: i) substance-specific transformation rates, ii) environmental conditions, iii) point of discharge (location of drug user) and iv) sewer network properties, primarily hydraulic residence time (HRT) and the ratio of biofilm contact area to wastewater volume $\left(A / V_{e q}\right)$.
\end{abstract}

Assessing associated uncertainties typically requires numerous simulations. Therefore, we propose a new two-step modeling framework: 1) Quantify hydrodynamic conditions. This computationally demanding step was performed once in SWMM to derive HRT and A/Veq for each potential point of discharge (node) in three catchments of different size. 2) Estimate biomarker loss. In this step, Monte

Carlo simulations were performed for defined scenarios. Depending on assumptions about drug user distribution and prevalence, a number of nodes was sampled. For each node an empirical first-order transformation model was applied with flow-path-corresponding $H R T$ and $A / V_{e q}$ from step 1 . Biotic and abiotic transformation rates were sampled from distributions combining variability of different biofilms.

In our modeling study, median losses were $>30 \%$ for amphetamine, 6-monoacetylmorphine and 6acetylcodeine in all three catchments with high uncertainty (5\% to $100 \%$ loss), which would imply a systematic underestimation of consumption when neglecting in-sewer processes. Median losses for 21 other investigated biomarkers were $<10 \%$ with different uncertainty ranges - "no substantial transformation" was confirmed for nine substances in a real sewer segment with a two-hour residence time. Transferability of these results must be tested for other catchments. Our approach allows efficient testing and, furthermore, can be expanded for many other human biomarkers. To further reduce uncertainty, mainly additional knowledge on transformation rates, particularly in biofilm, and their distribution across a sewer network is needed to update model input objectively. Accounting for 
biomarker stability during in-sewer transport will avoid biased estimates and further improve wastewater-based epidemiology.

\section{Key words.}

Wastewater quality modeling, micropollutant, Monte Carlo, sewage-based epidemiology, pharmaceuticals. 


\section{Nomenclature}

$A / V_{i} \quad\left[\mathrm{~m}^{-1}\right] \quad$ surface area of biofilm (wetted perimeter) to wastewater volume ratio in conduit $\mathrm{i}$

$A / V_{e q} \quad\left[\mathrm{~m}^{-1}\right] \quad$ HRT-normalized sum of all $\mathrm{A} / \mathrm{V}_{\mathrm{i}}$ along a flow path from input node to WWTP

HRT [h] Hydraulic residence time of water mass along a flow path

[PE] Population equivalents including/excluding industrial discharges

Residence time distribution of a tracer (dirac pulse over $4 \mathrm{~min}$ )

travel time

[h] Travel time $e_{i}$ within one conduit $\mathrm{i}$

60

\section{Graphical abstract}

Sewer network

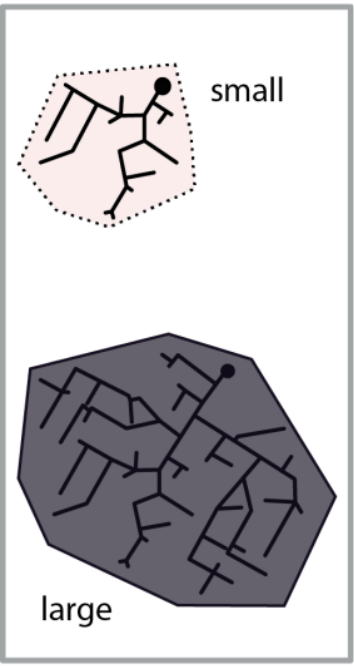

Variability in hydraulics

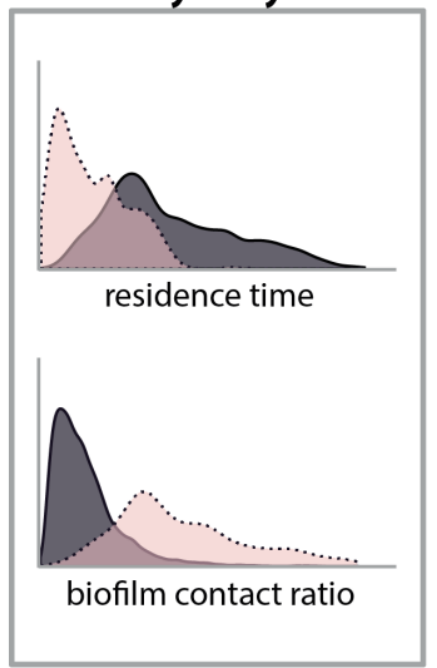

In-sewer biomarker transformation
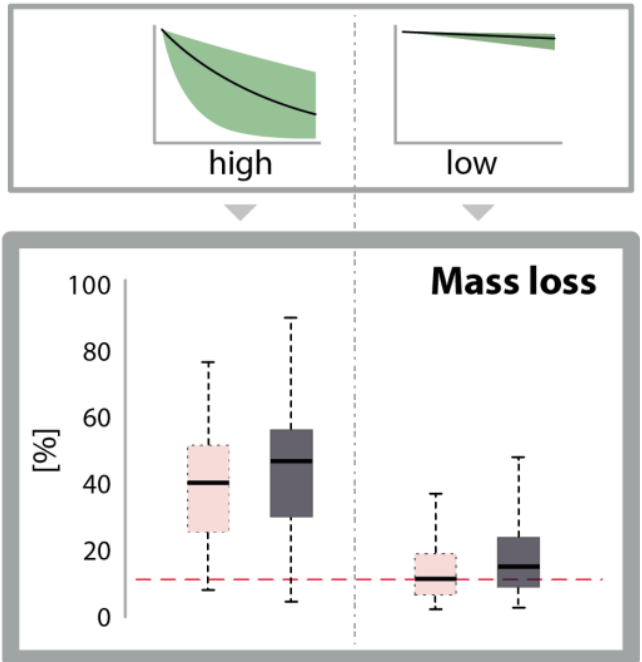


\section{Introduction}

Humans excrete trace amounts of substances such as pharmaceuticals or illicit drugs (subsequently referred to as biomarkers). Biomarkers in wastewater provide information about the excretion in a

catchment and are an indicator for consumption. The wastewater collection system unintendedly gathers this information and aggregates it in the inflow of wastewater treatment plants (WWTP). In wastewater-based epidemiology (WBE) selected biomarkers in this stream are used to estimate drug consumption at the community level (e.g. Ort et al., 2014). Currently, the unknown extent of in-sewer transformation in a specific catchment implies high uncertainty for such estimates. In-sewer transformation is studied in depth for conventional pollutants excreted by every person, e.g., ammonium, organic matter (Hvitved-Jacobsen et al., 2013; Nielsen et al., 1992). In contrast, few studies investigate biomarkers such as illicit drugs that are excreted only by a small percentage of the population in small amounts (McCall et al., 2016a). Under certain conditions, in-sewer processes can account for biomarker-specific transformation losses of up to $100 \%$ for e.g., 6-monoacetylmorphine and 6-acetylcodeine, influenced by sewer size, type and topology and environmental conditions (Jelic et al., 2015; McCall et al., 2016b; Ramin et al., 2016; Thai et al., 2014).

Therefore, to reliably apply WBE, each catchment should be evaluated for potential in-sewer biomarker losses due to transformation [we do not assess exfiltration and wet weather situations (e.g. losses via combined sewer overflows)]. Experimentally, this is difficult, since it would require i) spiking of deuterated-labeled biomarkers and a persistent tracer into one, of potentially thousands of toilets, ii) measuring biomarker and tracer residuals at the WWTP and iii) repeat this laborious procedure due to limited transferability of one experiment. Numerous experiments would be required to cover interand intra- catchment differences of environmental and hydraulic conditions, in both space (different cities, as well as different parts within a city) and time (seasonal and diurnal variability). 

quantified the uncertainty of the back-calculation including discharge measurements, chemical analysis and the applied sampling scheme but did not assess effects of temporal dynamics and spatial variation within the collection network. Including all sewer processes and degradation pathways with a stoichiometric matrix, as previously done in integrated urban water modeling of micropollutant fate (e.g., Snip et al., 2014), can result in a complex and over-parameterized approach in sewer networks (Vezzaro et al., 2014). So far, numerous studies modeled biomarker concentrations in sewer systems, mostly for the purpose to identify priority pollutants for environmental protection (Carballa et al., 2008; Daouk et al., 2016; Heberer and Feldmann, 2005). These studies described sewer networks as a homogeneous series of CSTRs or applied a travel time density function as an alternative to complex hydrodynamic models. To our knowledge, no study so far considered the influence of factors such as varying flow (e.g., velocity, fill level) on resulting conditions (e.g., HRT) influencing biomarker transformation processes. Sewer networks vary in size, topological structure and topography; wastewater composition and environmental conditions can be different. These factors influence the transformation processes and heavily depend on catchment characteristics so that effects on biomarker losses can a priori not be generalized. However, to date, an efficient approach to quantify the potential loss of biomarkers during conveyance in sewer systems considering network-specific characteristics and boundary conditions is lacking.

The objective of our work was to assess biomarker loss specifically during transport in sewers. Previous work showed that biofilm plays a role (McCall et al., 2016b). This implies that the wetted biofilm area must be determined for individual conduits, which requires a detailed hydraulic approach in view of flows varying over the day. Due to uncertain input, i.e. unknown location and distribution of drug users and variable transformation rates, there is a need to model several scenarios. Since computational efforts with existing hydrodynamic sewer modeling software are still considerable, we 
aim at identifying a fit-for-purpose water quality model to allow a quick assessment of an entire catchment at low computational costs. As inputs, such a model should consider biomarker-specific transformation rates and catchment-specific hydraulic information, specifically travel time of biomarkers from entry point to WWTP (hydraulic residence time along flow path, $H R T$ ) and the ratio of biofilm contact area to wastewater $(A / V)$ (McCall et al., 2016b). Spatio-temporal variability of wastewater flows affect travel time and $A / V$ in each conduit. To identify an efficient, yet suitable approach to determine realistic biomarker losses, including objective uncertainties, we had to:

(i) evaluate different levels of hydraulic model complexity,

(ii) describe and derive relevant hydraulic parameter distributions ( $H R T, A / V)$ considering effects of temporal (diurnal) and spatial variability (drug user location),

(iii) investigate different catchment scales to make an attempt to generalize results,

(iv) facilitate the evaluation of multiple biomarkers, numerous scenarios, environmental conditions and levels of prevalence (at low computational costs). 


\section{Material and methods}

To address the four points above in a modeling study (core of this work), we selected the sewer catchment of Dresden (Germany) implemented in EPA SWMM (see 2.5). It was thought to be of appropriate size (sufficiently long $H R T s$ ) so that transformation rates were expected to result in relevant losses for selected biomarkers. Additionally, to provide a plausibility check for observable losses in a real sewer, we performed a unique full-scale experiment. This was carried out in Zurich (Switzerland), in a five-kilometer sewer segment $(H R T=2 \mathrm{~h})$ without any lateral inflows (see 2.6).

\subsection{Conceptual approach}

We use the following transformation model to estimate biomarker loss (McCall et al., 2016b):

$$
\frac{d C_{\text {biomarker }}}{d t}=-\left(k_{a}+k_{W W}+k_{\text {biofilm }} \frac{A}{V}\right) C_{\text {biomarker }}
$$

where $\mathrm{C}_{\text {biomarker }}$ is the concentration of the targeted illicit drug biomarker [mol L-1], $k_{a}$ is the abiotic transformation rate coefficient $\left[\mathrm{h}^{-1}\right], k_{W W}$ is the biotransformation rate coefficient in the bulk liquid $\left[\mathrm{h}^{-}\right.$ ${ }^{1}$ ], and $k_{\text {biofilm }}$ is the biotransformation rate coefficient for biofilm processes $\left[\mathrm{m} \mathrm{h}^{-1}\right]$. The ratio of the biofilm surface area $A$ (calculated as the wetted perimeter) and the wastewater volume $V\left(A / V_{i}\left[\mathrm{~m}^{2} \mathrm{~m}^{-}\right.\right.$ ${ }^{3}$ ]) normalizes $k_{\text {biofilm, }}$, to consider different fill levels and corresponding biofilm contact ratios (different cross sections, diurnal variations), assuming $A / V_{i}$ is constant along each conduit $i$.

In each conduit $i$, resulting travel time $i$ and $A / V_{i}$ follow a diurnal profile similar to the pattern of the dry weather inflow. To account for both spatial and temporal variability of hydraulic parameters, a set of transport and quality simulations needs to be run for each biomarker, each time point and each location of potential point of discharge. Current modeling approaches - available for both, hydraulic, as well as water quality modeling in sewers - demand high computational effort for a single run since they simulate biomarker concentrations in each conduit for each time point. In view of the anticipated 
high number of runs needed to calculate different scenarios for (unknown) drug user locations and uncertain rate coefficients this would imply enormous computational efforts. Therefore, we decoupled transport and quality calculations. From the hydraulic calculations (for different levels of detail), we store velocities and fill levels for each time step and conduit. From this information, we assign each node (i.e. a potential location of drug users) a flow path with a representative $H R T$ (sum of travel times along the flow path). To account for variability of $A / V_{i}$ along the flow path, we calculated $A / V_{e q}$ as the sum of the $A / V_{i}$ of each conduit $i$ weighed with the residence time of conduit $i\left(H R T_{i}\right)$ :

$$
A / V_{e q}=\frac{\sum_{i} H R T_{i} \cdot \frac{A}{V_{i}}}{\sum_{i} H R T_{i}}
$$

Our approach was to stochastically simulate biomarker loss for real-life sewer catchments of varying scale and different conditions. A Monte Carlo framework was applied to estimate the potential range of biomarker loss with the following input factors: i) transformation processes in biofilm and wastewater $\left(k_{\text {biofilm }}, k_{a}\right.$ and $k_{W W}$; see section 2.4$)$ and ii) catchment-specific hydraulic parameters (HRT and $A / V_{\text {eq; }}$ see section 2.2). This procedure was applied for different scenarios $S$ (see section 2.3).

For each scenario 3,000 runs were simulated, to provide stable distributions of results. In each run, new nodes were sampled with the number of nodes depending on prevalence of drug use (except scenario S3 and S4, see Table 2). Within one run only one value for each $k_{a}, k_{w w}$, and $k_{b i f f i m}$ was sampled and applied to all flow paths in the entire catchment. This was done due to the following reason. If for each conduit or flow path a different value was sampled, the effect would be that a transformation rate close to average would result for the entire catchment in each run.

In a sensitivity analysis we assessed how input variability affected resulting distributions. This was done by assuming constant values for i) transformation rates, ii) $A / V_{e q .}$ for all runs in a scenario. 


\subsection{Representation of hydraulic variability}

Instead of implementing the transformation model (equation 1) directly in a hydrodynamic water quality modeling tool (subsequently considered as approach A), we applied approaches of varying levels of detail to derive frequency distributions of $H R T$ (see Table 1).

Table 1. Conceptual overview of different approaches taken to investigate different levels of detail in sewer catchment modeling.

\begin{tabular}{|c|c|c|c|c|}
\hline & A & $\mathrm{B}-\mathrm{HRT}_{\text {disp }}$ & $\mathrm{C}-\mathrm{HRT}_{\mathrm{adv}}$ & $\mathrm{D}$ \\
\hline & $\begin{array}{l}\text { Transport and quality } \\
\text { simulation }\end{array}$ & Transport simulation & $\begin{array}{l}\text { Hydraulic simulation } \\
\text { (without tracer) }\end{array}$ & $\begin{array}{l}\text { Expert Estimate (local } \\
\text { sewer operator) }\end{array}$ \\
\hline $\begin{array}{l}\text { Processes } \\
\text { considered }\end{array}$ & $\begin{array}{l}\text { Hydraulic software (e.g., } \\
\text { SWMM) } \\
\text { Advection, dispersion, } \\
\text { reaction (quality) }\end{array}$ & Advection, dispersion, & Advection & $\begin{array}{l}\text { experience (e.g., } \\
\text { qualified guess, tracer } \\
\text { experiment, hydraulic } \\
\text { simulation) }\end{array}$ \\
\hline
\end{tabular}

For these approaches $B, C$ and $D$ we modeled each flow path as a single plug flow reactor in which we applied biomarker transformation kinetics adapted from eq. 1:

$$
M=M_{0} \exp \left[-H R T\left(k_{a}+k_{W W}+k_{\text {biofilm }} \frac{A}{V_{e q}}\right)\right]
$$

where the change in biomarker mass from entry point $\left(M_{0}\right)$ to WWTP $(M)$ is obtained from the rate coefficients and the path-specific $H R T$ and $A / V_{\text {eq. }}$

In approach $B$, the frequency distributions of HRTs were generated with a transport simulation. At each node a different tracer was added in the SWMM model at different times of the day $(2 \mathrm{am}, 8 \mathrm{am}$, $2 \mathrm{pm}, 8 \mathrm{pm}$ ) to assess temporal variability of $H R T$ due to the diurnal variation of wastewater flows. Then the normalized mass load at the WWTP was calculated to obtain a residence time distribution $(R T D)$. The mean values of the RTDs are used as node- and time-specific HRTs. This method accounted for both dispersion and advection of the tracer (inert biomarker) along the flow path. In approach $C$, the spatial distributions of $H R T$ was calculated from velocities and water levels in each conduit along the flow path, thus neglecting dispersion phenomena. The flow path from each node 
through the relevant conduits to the WWTP was identified with the Dijkstra algorithm on a directed graph extrapolated from the sewer model (Csardi and Nepusz, 2006). Distributions for the $A / V_{\text {eq }}$ for all nodes (all flow paths) were calculated based on approach $C$.

In approach $D$, the best estimate for the average $H R T$ relied on an informed expert guess. The accuracy of this information can vary highly and is difficult to assess. We assumed that spatial variation of $H R T$ follows a normal distribution using the expert guess as mean and assuming a relative standard deviation of $25 \%$.

\subsection{Uncertainties due to prevalence and environmental conditions}

In five scenarios we assessed the effect on predicted biomarker loss caused by different assumptions on

i) distributions of drug user locations, ii) levels of prevalence of drug use and iii) wastewater temperature (Table 2). In scenario S1 and S2, drug users were assumed to be randomly distributed in the catchment (weighted with population per node). 
Table 2. Selected scenarios to evaluated effect of biomarker loss. Please note: in our modeling study, each drug user discharges the same amount of drug (transformation rates are expected to be independent within a reasonable concentration range). Prevalence defines the number of drug users and hence the number of nodes to be selected (see also 2.1).

\begin{tabular}{|l|l|l|l|l|l|}
\hline Scenarios & $\mathbf{S 1}_{1 \%}$ & $\mathbf{S 2}_{\mathbf{0 . 0 1} \%}$ & $\mathbf{S 2}_{\text {temp }}$ & $\mathbf{S 3}_{\text {shortHRT }}$ & $\mathbf{S 4}_{\text {longHRT }}$ \\
\hline $\begin{array}{l}\text { Prevalence (\% of population consuming } \\
\text { drug on a particular day) }\end{array}$ & $1 \%$ & $0.01 \%$ & \multicolumn{2}{|l|}{} \\
\hline Drug user location & \multicolumn{3}{|l|}{} & $\begin{array}{l}\text { closest flow } \\
\text { distance to } \\
\text { sampling } \\
\text { location }\end{array}$ & $\begin{array}{l}\text { longest flow } \\
\text { distance } \\
\text { from } \\
\text { sampling } \\
\text { location }\end{array}$ \\
\hline Transformation rates & randomly distributed in the catchment & $\begin{array}{l}\text { Rates halved } \\
\text { (temperature } \\
\left.10^{\circ} \mathrm{C}\right)\end{array}$ & Temperature $22^{\circ} \mathrm{C}$ \\
\hline
\end{tabular}

In two extreme scenarios we assumed, we would know that all drug users discharged close to the

WWTP $\left(\mathrm{S} 3_{\text {shortHRT }}\right)$ or far away $\left(\mathrm{S} 4_{\text {longHRT }}\right)$. We selected two representative nodes and calculated the biomarker loss with corresponding $H R T$ and $A / V_{\text {eq. }}$. To account for varying prevalence of drug use and investigate the effect on uncertainty we tested a scenario with low prevalence $(0.01 \%$ of total population are drug users on a particular day, S2), and high prevalence (1\%, S1). The effect of temperature on transformation rates was also considered ( $\left.\mathrm{S} 2_{\text {temp }}\right)$. In the absence of reliable 215 information on redox conditions for both, spatial distribution in the catchments and effect on transformation rates, we assumed aerobic conditions in the bulk liquid and biofilm surface (dissolved oxygen $>2 \mathrm{mg} \mathrm{L}^{-1}$ ) for all pipes in the gravity sewer.

\subsection{In-sewer transformation rates}

Transformation of biomarkers through biofilms can vary from conduit to conduit (McCall et al., 2016).

Since we had no information about transformation rates for biofilms in Dresden, we used literature data. Applying Bayesian inference, transformation rate coefficients for $k_{b i o f i l m}$ (and $k_{W W}$ ) were estimated from previously conducted batch experiments with biofilms from four different gravity sewers in Switzerland (McCall et al., 2016b; tested under aerobic conditions, $22^{\circ} \mathrm{C}, \mathrm{pH} 7-8$; see Supporting Information SI2 for detailed information). The resulting rate coefficient $k_{b i f f i m}$ provides a measure of 
the natural variability in transformations for biofilms in different sewer stretches and from different locations. To quantify this variability the model was extended by the assumption that the distribution of all biofilm rate coefficients are gamma distributed. This model provides the advantage that it can easily be updated when new experimental data becomes available.

\subsection{Sewer catchment and hydraulic model implementation}

The SWMM model of the sewer network of the city of Dresden (Germany) was used to exemplary assess biomarker transformations. It was validated for dry weather flow conditions using continuous flow data collected at several locations (see SI3 and Karpf and Krebs, 2011 for more details). We investigated a small, medium and large catchment (see Figure 1). The large one is a substantial part of the entire Dresden sewer catchment (population 370,000 p, longest flow path $24 \mathrm{~km}$ ). The Dresden sewer catchment can be physically separated into two main sub-catchments, both discharging into the same central large WWTP. The smaller of the two sub-catchments was investigated as our medium catchment $(90,000 \mathrm{p}, 16 \mathrm{~km})$. As the small catchment we considered a small sub-catchment $(14,000 \mathrm{p}$, $9 \mathrm{~km}$; with a virtual sampling point/WWTP). The a priori estimated average $H R T$ in the large catchment is about $6 \mathrm{~h}$ based on the diurnal inflow regime at the central WWTP and day-to-day observations of the sewer network operator (qualified guess). For the medium and small catchments we assumed values of $4 \mathrm{~h}$ and $2 \mathrm{~h}$, respectively. The $H R T$ distributions were obtained by assuming a normal distribution $($ mean $=$ expert guess; $s d=25 \%)$. 


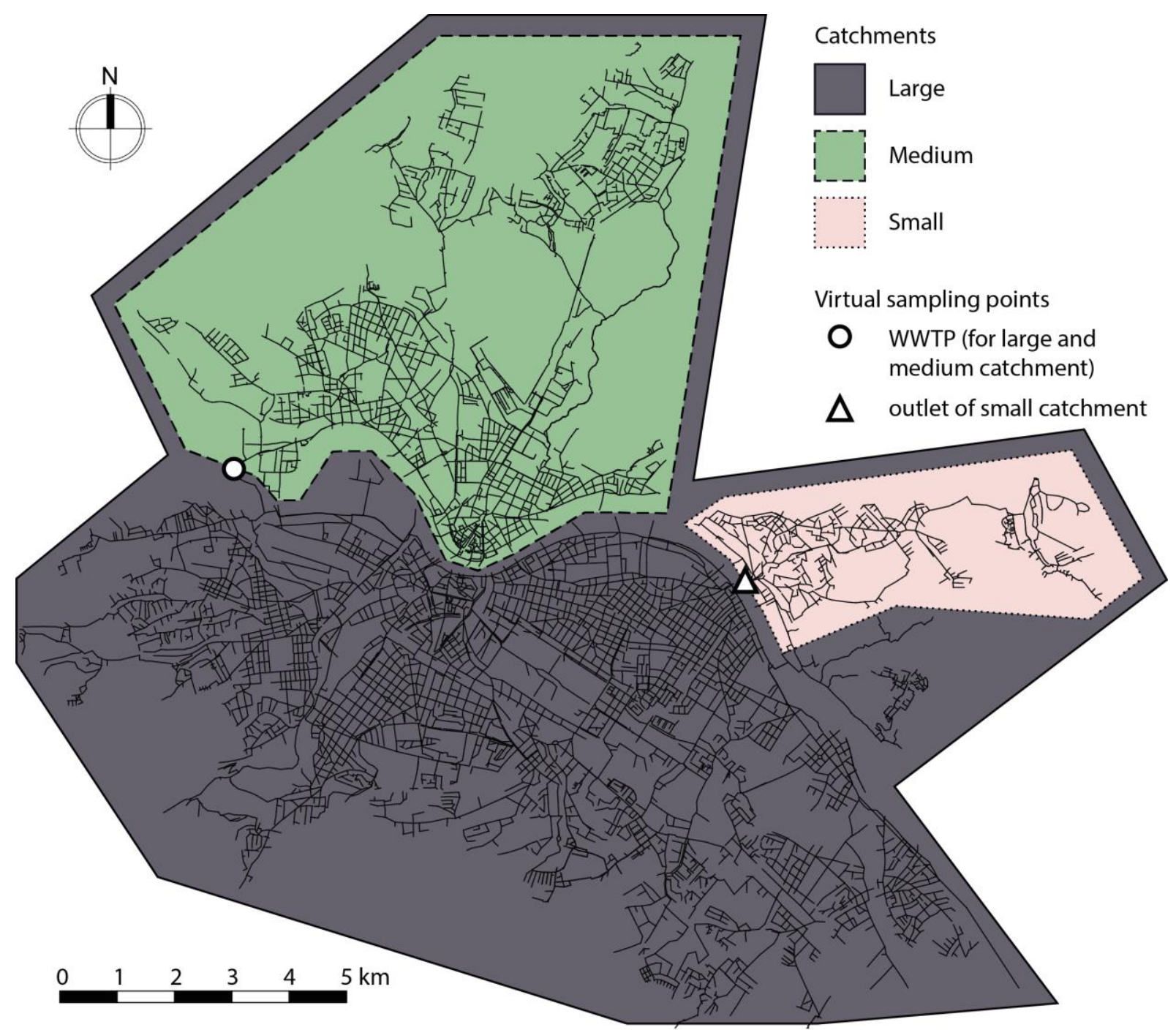

Figure 1. Representation of Dresden's sewer network with conduits implemented in SWMM, boundaries of three (sub)catchments and virtual sampling points (most downstream point in each catchments).

For hydraulic simulations in approach B and C, we used the open source software EPA-SWMM v. 5.1.011 (Rossman, 2010). SWMM calculates the mixing and transport in each conduit, modeling each as a continuously stirred tank reactor and solves the full dynamic Saint Venant differential equations to estimate mass transport. Each hydrodynamic simulation is run with a 20 -second routing step time. Data preparation and visualization were performed with R (R-Core-Team, 2015).

Any other sewer network of similar size could have been used to illustrate the application of the proposed method. The Dresden case was primarily selected because of i) size (sufficiently long HRTs) and ii) the availability of a validated sewer model. While the Dresden sewer model was implemented in 
SWMM, any other software that allows retrieving the relevant hydraulic characteristics (see 3.2) can be used.

\subsection{Experimental full-scale study on in-sewer transformation}

It is very laborious to monitor biomarkers in a real sewer over a sufficiently long distance (HRT) to quantify biomarker transformation reliably. This is mainly for two reasons: i) either large quantities of labeled biomarkers would have to be spiked into a sewer at an upstream location (including an inert tracer to quantify losses due to unknown leakage and compensate for potential flow measurement errors) with subsequent analysis of a representative downstream sample or ii) a transport sewer segment with no lateral confluents and sufficiently long HRT must be available to observe losses of biomarkers naturally present in the wastewater in a conceptually simple input-output comparison.

In Zurich (Switzerland) we had the opportunity to assess in-sewer transformation in the five-kilometer and with neither in- nor exfiltration. Dry weather flow fluctuates between 200 and $800 \mathrm{~L} \mathrm{~s}^{-1}$ resulting in variable residence times. The intermittent operation of the two pipes, switching flow every $24 \mathrm{~h}$, implies that there is only wastewater in one of the two pipes during dry weather and the biofilm never dries out (for more information see Kaegi et al., 2013). (at $2400 \mathrm{~m}$ ). For our experiment we diverted a constant flow of $30 \mathrm{~L} \mathrm{~s}^{-1}$ into the empty pipe, in which a relevant residence time of $2 \mathrm{~h}$ resulted between $\mathrm{L} 1$ and $\mathrm{L} 3$ with an $\mathrm{A} / \mathrm{V}$ ratio of $11 \mathrm{~m}^{-1}$. The constant flow allowed collecting representative samples at L1 and L3 in a time-proportional manner (no flow signal needed). An additional unique feature of this sewer is that it is cleaned every five years, which allowed the evaluation of transformation with and without biofilm in full scale, which is quite seldom. 
Five years after the last cleaning of the sewer pipes, our first sampling campaign was on 8 January 2015 with thick, mature biofilm covering the usually submerged parts of the sewer walls. The second sampling campaign started on 19 March 2015. This was three days after several weeks of high-pressure cleaning the smooth stonewear pipe surface with a specialized machine and near-complete removal of the biofilm. In the first sampling campaign a small, continuous side-stream was diverted into $10 \mathrm{~L}$ Schott bottles in an insulated container with dry ice $\left(<4^{\circ} \mathrm{C}\right.$; peristaltic pump Heidolph Pumpdrive 5001, $10 \mathrm{rpm}$; hose bore tube $3.2 \mathrm{~mm}$ ). Due to unexpected, repeated clogging of sample hoses in the second campaign, two autosampler were installed at L1 and L3 to collect back-up samples (Sigma 900, PE bottles, sampling frequency $\mathrm{dt}=2 \mathrm{~min}$ ). Triplicates were analyzed with a previously validated method (McCall et. al, 2016b).

\section{Results}

We first present biomarker transformation rates as half-lives (3.1). For three catchments of different size, we then compared hydraulic characteristics $(H R T)$ obtained with different approaches, i.e. levels of detail (3.2), and combined results to estimate biomarker losses including uncertainty assessment and sensitivity analysis (3.3).

\subsection{Transformation rates}

To illustrate the biomarker-specific, individual influences of $k_{a}, k_{W W}$ and $k_{b i o f i l m}$ on the overall in-sewer process rate, the rate coefficients were expressed individually as half-lives $\left(t_{0.5}\right)$. Based on equation 1 , the combined effects (as $t_{0.5}$ ) of all rate coefficients were demonstrate, using a range of realistic $A / V_{e q}$ ratios of $17 \mathrm{~m}^{-1}, 33 \mathrm{~m}^{-1}$, and $66 \mathrm{~m}^{-1}$ (Table 3, rate coefficients with 10\%- and 90\%-quantiles can be found in SI4). The half-lives show how abiotic $\left(k_{a}\right)$, wastewater $\left(k_{w w}\right)$ and biofilm processes $\left(k_{b i o f i l m}\right)$ affect the stability of biomarkers under aerobic conditions $\left(22^{\circ} \mathrm{C}, \mathrm{pH} 7-8\right)$. 
Table 3. Mean half-life ( $\left.\mathrm{t}_{0.5}\right)$ for biomarkers based on abiotic-, wastewater- and biofilm-induced in-sewer transformations (gravity sewer conditions: aerobic, $22^{\circ} \mathrm{C}, \mathrm{pH} 7-8$ ). $\mathrm{k}_{\text {overall }}$ is the $\mathrm{t}_{0.5}$ aggregating the three individual mean rate coefficients with different surface area biofilm to wastewater ratios $\left(\mathrm{A} / \mathrm{V}\left[\mathrm{m}^{-1}\right]\right)$. Red: half-lives $<12 \mathrm{~h}$ [mean HRT of many large catchments is $<12 \mathrm{~h}$, see SI of Ort et al., 2014 ( $n=32$ cities; $\min =1 \mathrm{~h}, \max =12 \mathrm{~h}$, mean=5.5 h)]. Green (italic): names of biomarkers that showed $<20 \%$ loss over $24 \mathrm{~h}$ with A/Vs up to $66 \mathrm{~m}^{-1}$ in previous batch experiments for all tested biofilms (McCall et al., 2016b).

\begin{tabular}{|c|c|c|c|c|c|c|c|c|c|}
\hline \multirow{2}{*}{\multicolumn{2}{|c|}{ Half-lives $t_{0.5}$ [hour] }} & \multicolumn{5}{|c|}{ considered process } & \multicolumn{3}{|c|}{ Overall transformation } \\
\hline & & & \multirow{2}{*}{$\frac{\mathbf{k}_{\mathrm{ww}}}{54}$} & \multirow{2}{*}{ 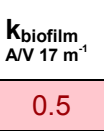 } & \multirow{2}{*}{ 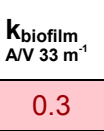 } & \multirow{2}{*}{$\begin{array}{c}\frac{\mathbf{k}_{\text {biofilm }}}{{\text { Av } 66 \mathrm{~m}^{-1}}^{-1}} \\
0.1\end{array}$} & \multirow{2}{*}{$\begin{array}{c}\mathbf{K}_{\text {overall }} \\
\text { Av } 17 \mathbf{~ m}^{-1}\end{array}$} & \multirow{2}{*}{$\begin{array}{c}\begin{array}{l}k_{\text {overall }} \\
\text { A } \mathbf{3} \mathbf{3 3} \mathbf{m}^{-1}\end{array} \\
0.3 \\
\end{array}$} & \multirow{2}{*}{$\begin{array}{c}\begin{array}{c}\mathbf{K}_{\text {overall }} \\
\text { A/V } 66 \mathrm{~m}^{-1}\end{array} \\
0.1\end{array}$} \\
\hline$A C$ & 6-acetylcodeine & & & & & & & & \\
\hline AMP & amphetamine & 150 & 113 & 9 & 5 & 2.3 & 8 & 4 & 2.2 \\
\hline $\mathrm{BE}$ & benzoylecgonine & 1185 & 1567 & 72 & 37 & 19 & 65 & 35 & 18 \\
\hline$C B Z$ & carbamazepine & 1821 & 3801 & 610 & 314 & 157 & 408 & 250 & 139 \\
\hline COC & cocaine & 20 & 101 & 58 & 30 & 15 & 13 & 11 & 8 \\
\hline COE & cocaethylene & 35 & 181 & 45 & 23 & 12 & 18 & 13 & 8 \\
\hline$D C F$ & diclofenac & 1443 & 3417 & 330 & 170 & 85 & 249 & 146 & 78 \\
\hline EDDP & $\begin{array}{l}\text { 2-ethylidene-1,5-dimethyl-3,3- } \\
\text { diphenylpyrrolidine }\end{array}$ & 251 & 756 & 351 & 181 & 90 & 123 & 92 & 61 \\
\hline HMMA & 4-hydroxy-3-methoxymethamphetamine & 393 & 21 & 77 & 40 & 20 & 16 & 13 & 10 \\
\hline KET & ketamine & 695 & 1553 & 374 & 193 & 96 & 210 & 137 & 80 \\
\hline MAM & 6-monoacetylmorphine & 72 & 70 & 3 & 1.5 & 0.8 & 3 & 1.4 & 0.7 \\
\hline MDMA & 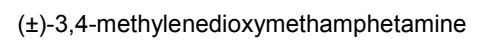 & 1029 & 1754 & 156 & 80 & 40 & 126 & 71 & 38 \\
\hline MDPV & methylenedioxypyrovalerone & 270 & 703 & 260 & 134 & 67 & 111 & 79 & 50 \\
\hline MEPH & mephedrone & 18 & 392 & 307 & 158 & 79 & 16 & 16 & 14 \\
\hline METH & methamphetamine & 745 & 663 & 236 & 122 & 61 & 141 & 90 & 52 \\
\hline MPA & methiopropamine & 1296 & 2381 & 156 & 80 & 40 & 131 & 73 & 38 \\
\hline MTO & methoxetamine & 830 & 2212 & 943 & 486 & 243 & 368 & 269 & 173 \\
\hline NorCOC & norcocaine & 14 & 25 & 106 & 54 & 27 & 8 & 8 & 7 \\
\hline NorKET & norketamine & 1113 & 32 & 945 & 487 & 243 & 31 & 30 & 28 \\
\hline ODMT & O-desmethyltramadol & 473 & 34 & 1108 & 571 & 285 & 31 & 30 & 28 \\
\hline PMA & 4-methoxyamphetamine & 167 & 220 & 102 & 53 & 26 & 49 & 34 & 21 \\
\hline PMMA & methoxymethamphetamine & 835 & 1367 & 176 & 91 & 45 & 131 & 77 & 42 \\
\hline TRA & tramadol & 1326 & 2918 & 751 & 387 & 193 & 412 & 272 & 160 \\
\hline$Z O L$ & zolpidem & 513 & 805 & 310 & 160 & 80 & 156 & 106 & 64 \\
\hline
\end{tabular}




\subsection{Hydraulic catchment characterization}

Results from the SWMM simulations for the approaches to describe hydraulic properties with different levels of detail (Table 1) are presented as frequency distributions. Spatial variability of $H R T_{a d v}$ was calculated as daily average of all flow conditions (Figure 2). Although, temporal variability in individual conduits can be considerable, the effect for biomarker loss calculations over an entire flow path seems small (see discussion in 4.2.1). The mean $H R T_{\text {disp }}$ and $H R T_{a d v}$ differ $<4 \%$ for all three catchment scales except the 10\%-quantile for the small and medium catchment ( $12 \%$ and $-45 \%$ ) (Table

315 4; Figure S5 in SI6). Frequency distributions for $A / V_{\text {eq }}$ were similar for the medium and large catchments with average values around $30 \mathrm{~m}^{-1}$ while the mean value for the small network was $98 \mathrm{~m}^{-1}$ (Figure 3). 

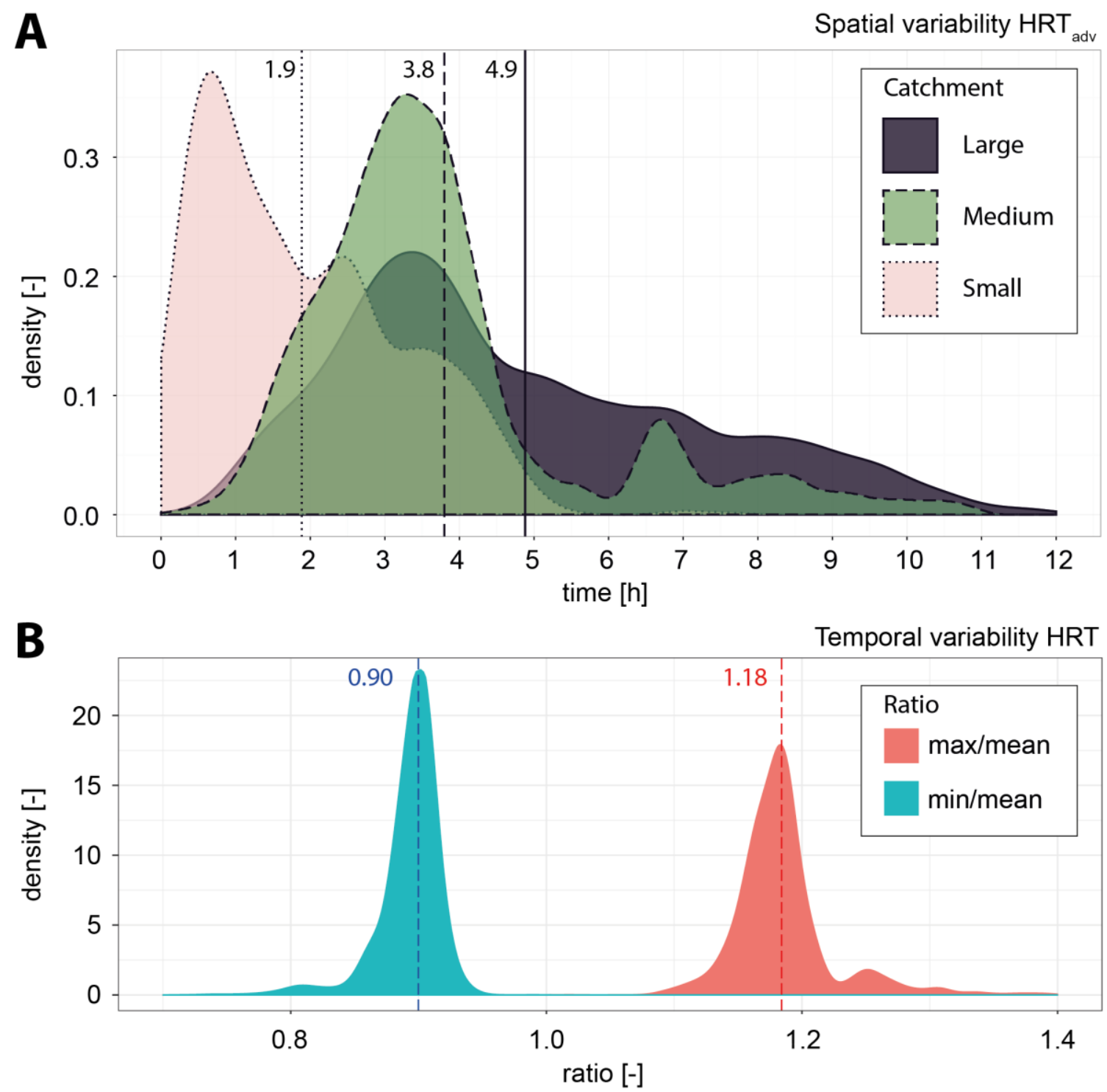

Figure 2. A: Frequency distributions for spatial variability of $\mathrm{HRT}_{\text {adv. }}$ B: Temporal variability of HRT as ratio of extreme daily minimum and maximum conditions divided by daily average conditions $\mathrm{HRT}_{\min } / \mathrm{HRT}_{\text {mean }}$ and $\mathrm{HRT}_{\max } / \mathrm{HRT}_{\text {mean }}$ for the large catchment. A+B: Each flow path once, i.e. not weighted with population; vertical lines represent distribution means. 
A

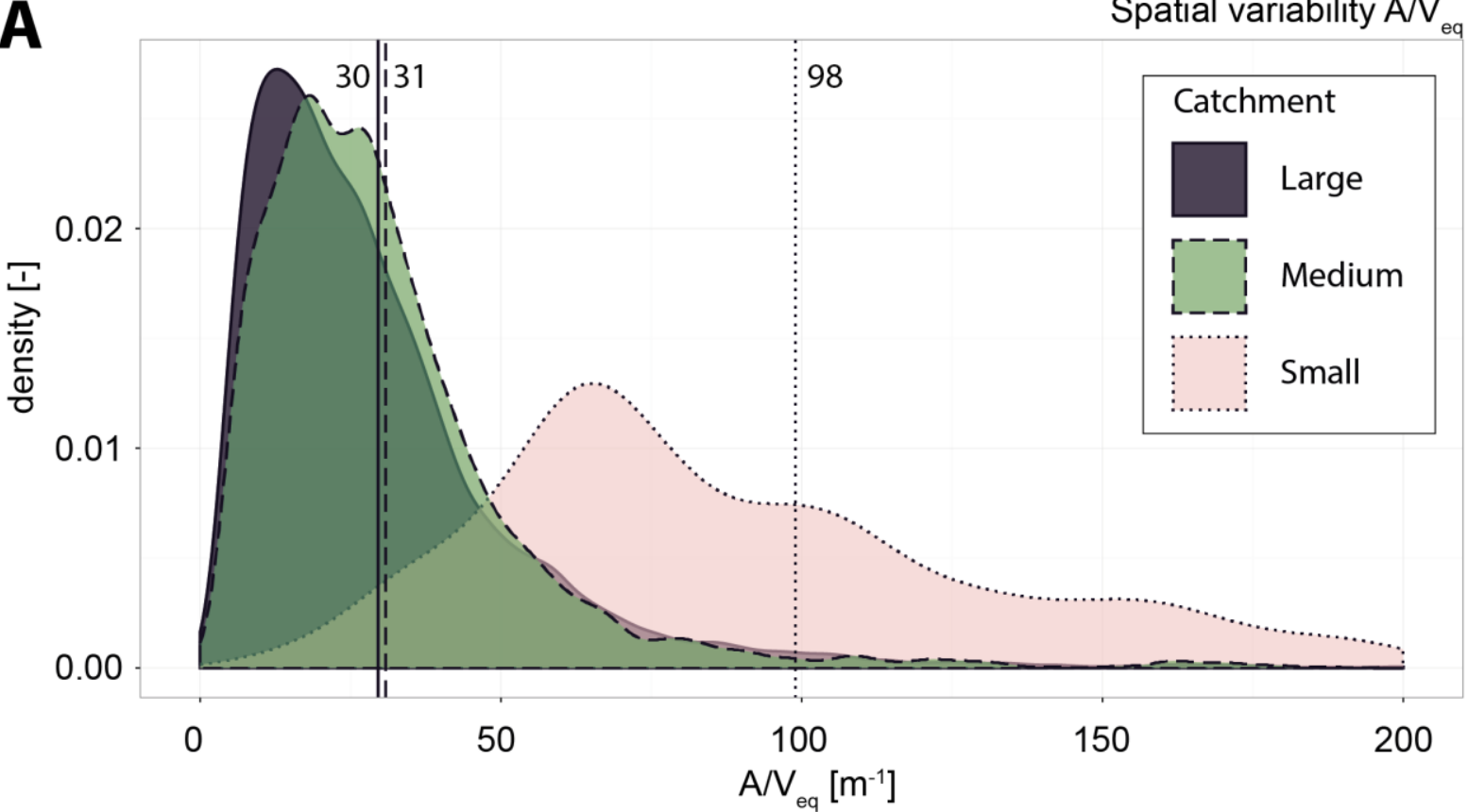

B

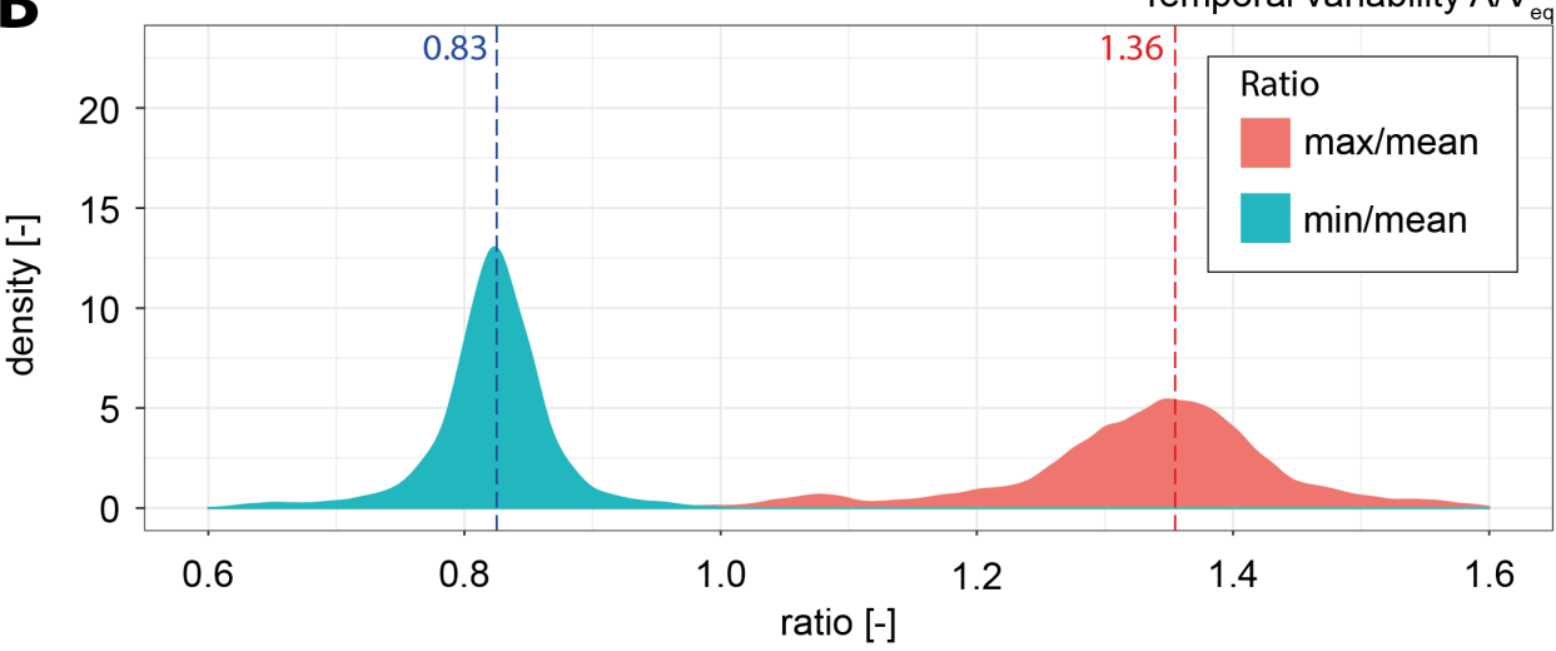

Figure 3. Frequency distributions for spatial variability of $A / V_{\text {eq. }}$ B: Temporal variability of $H R T$ as ratio of extreme daily minimum and maximum conditions divided by daily average conditions $\mathrm{A} / \mathrm{V}_{\text {eq,min }} / \mathrm{A} / \mathrm{V}_{\text {eq,mean }}$ and $\mathrm{A} / \mathrm{V}_{\text {eq,max }} / \mathrm{A} / \mathrm{V}_{\text {eq,mean }}$ for the large catchment. A+B: Each flow path once, i.e. not weighted with population; vertical lines represent distribution means.

Table 4. Summary statistics of the spatial frequency distributions of the small, medium and large catchments.

\begin{tabular}{|c|c|c|c|c|c|c|c|c|c|c|c|c|}
\hline \multirow[t]{2}{*}{ Catchment } & \multicolumn{4}{|c|}{ small } & \multicolumn{4}{|c|}{ medium } & \multicolumn{4}{|c|}{ large } \\
\hline & q10\% & mean & median & q90\% & q10\% & mean & median & q90\% & q10\% & mean & median & q90\% \\
\hline $\mathrm{HRT}_{\text {adv }}[\mathrm{h}]$ & 0.41 & 1.89 & 1.58 & 3.89 & 1.90 & 3.80 & 3.37 & 6.77 & 2.11 & 4.88 & 4.19 & 8.57 \\
\hline $\mathrm{HRT}_{\text {disp }}[\mathrm{h}]$ & 0.46 & 1.91 & 1.67 & 3.85 & 1.96 & 3.94 & 3.52 & 6.83 & 2.19 & 4.97 & 4.36 & 8.64 \\
\hline
\end{tabular}



nodes to the WWTP. On the contrary, the outer nodes had often a higher $A / V_{\text {eq }}$ (irrespective of distance to WWTP; Figure 4).

A
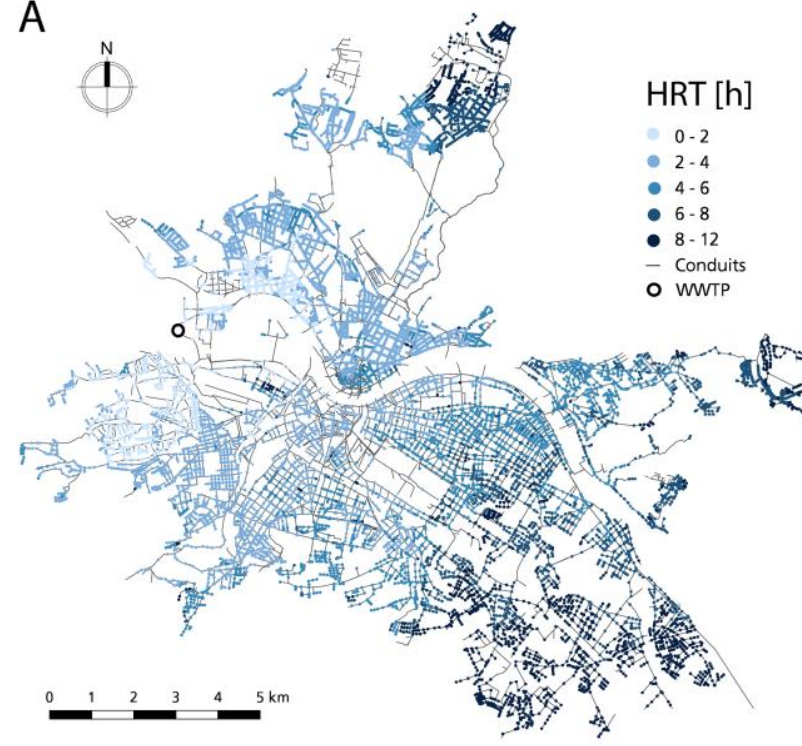

B
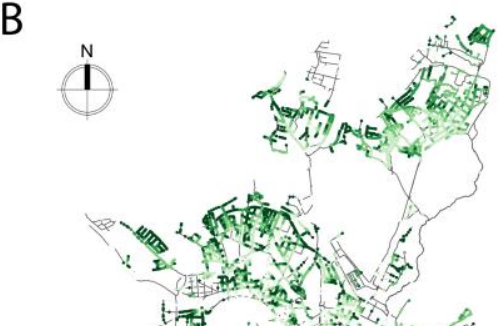

$$
\begin{aligned}
& \mathrm{A} / \mathrm{V}_{\text {eq }}\left[\mathrm{m}^{-1}\right] \\
& 0-10 \\
& 10-20 \\
& 20-30 \\
& -30-40 \\
& -40-100 \\
& - \text { Conduits } \\
& \text { - WWTP }
\end{aligned}
$$
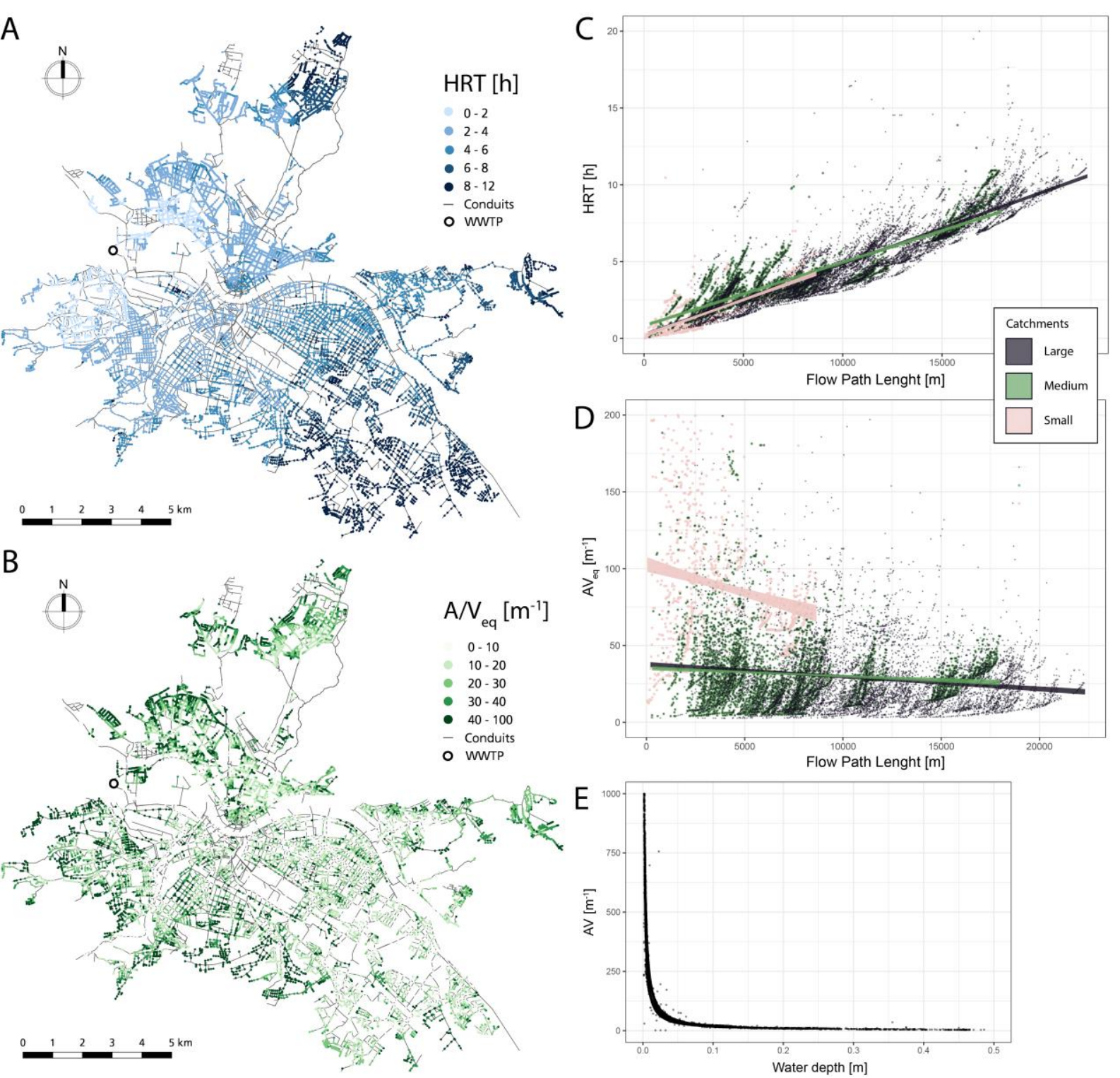

Figure 4. Spatial variability of $\operatorname{HRT}_{\text {adv }}(\mathbf{A})$ and $\mathrm{A} / \mathrm{V}_{\mathrm{eq}}(\mathbf{B})$ for the large catchment. C: Flow-path-specific HRTadv vs. total path length from node to wastewater treatment plant. D: Flow-path-specific $A / \mathrm{V}_{\text {eq }}$ vs. total path length. E: Water depth in a conduit and resulting A/V. - All calculations were based on daily mean flow conditions (neglecting temporal variability and dispersion; approach C). 


\subsection{Biomarker loss calculations}

For three biomarkers with high abiotic rates $k_{a}(\mathrm{COC}, \mathrm{NorCOC}, \mathrm{MEPH})$ a mass loss between $10 \%$ and $25 \%$ (median) was observed in all catchments for scenario S2 with $0.01 \%$ prevalence of drug use in the population (Figure 5; Figure 6; Figure S8 in the SI7). Although, loss was higher for these three biomarkers in the large catchment (Figure 6), biomarkers with comparably high $k_{\text {biofim }}$ (AMP, MAM, AC) had transformation losses ranging from $30 \%$ to $90 \%$ (median) with higher median losses in the small catchment. All other biomarkers showed $<10 \%$ loss in all catchments and tested scenarios. The effects of prevalence, temperature, uncertainty of transformation rates, location of users, and expert estimate are shown in Figure 7 for AMP and MEPH, two substances with different stability.

A previous study showed that temperature is inversely proportional to the biotransformation rate coefficient and rate coefficients (Suárez et al., 2012). The rate coefficients we applied in our study were determined from experiments conducted at $22{ }^{\circ} \mathrm{C}$. Therefore, in scenario $\mathrm{S} 2_{\text {temp }}$, rate coefficients were halved assuming lower wastewater temperatures around $10^{\circ} \mathrm{C}$. As a consequence, median biomarker loss was approx. $5 \%$ to $20 \%$ lower (Figure 7 ).

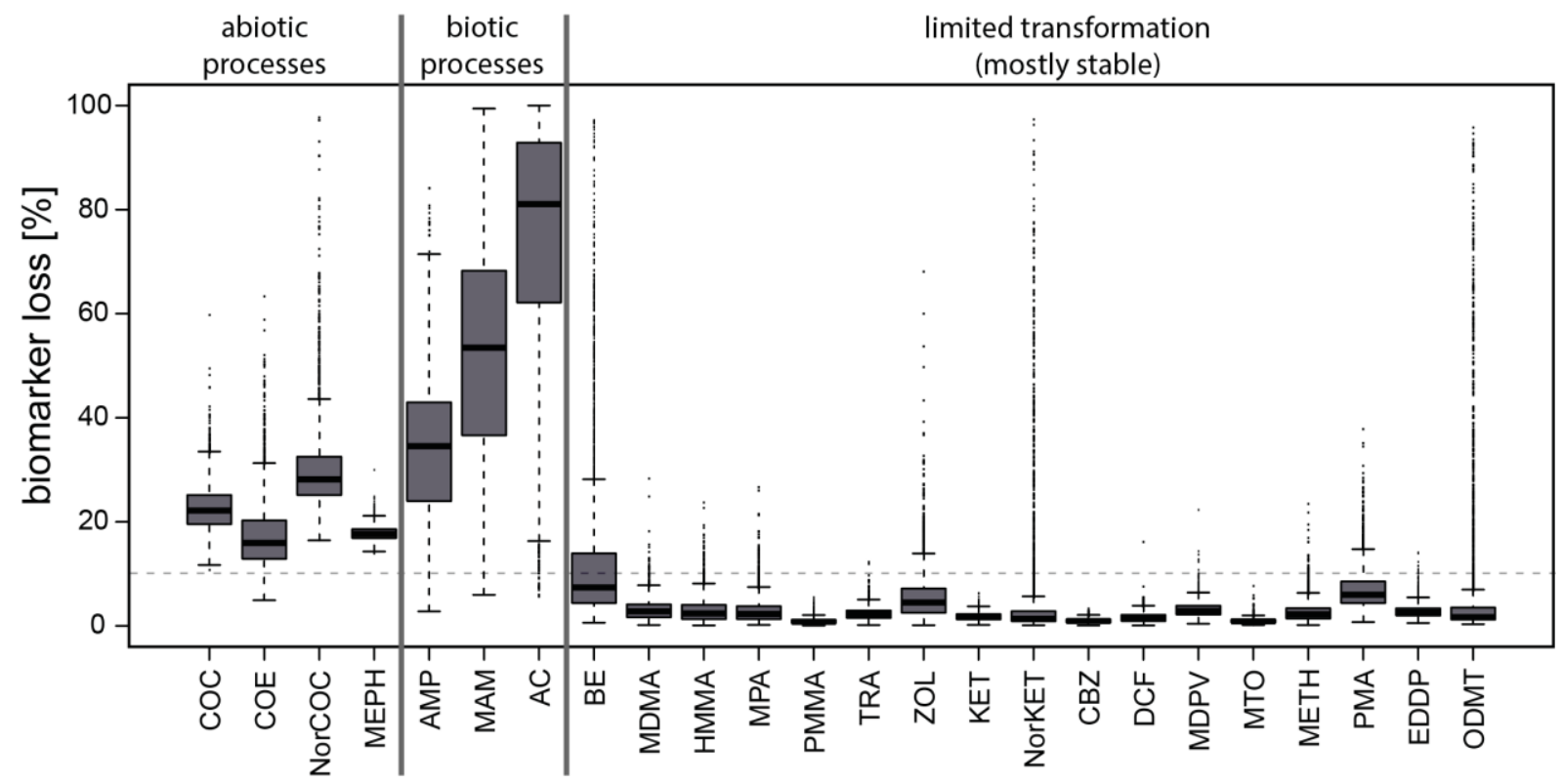

355 Figure 5. In-sewer transformation losses in the large catchment (scenario S2) calculated for daily mean flow conditions (neglecting temporal variability and dispersion; approach C). Dashed horizontal line indicates $10 \%$ loss. 


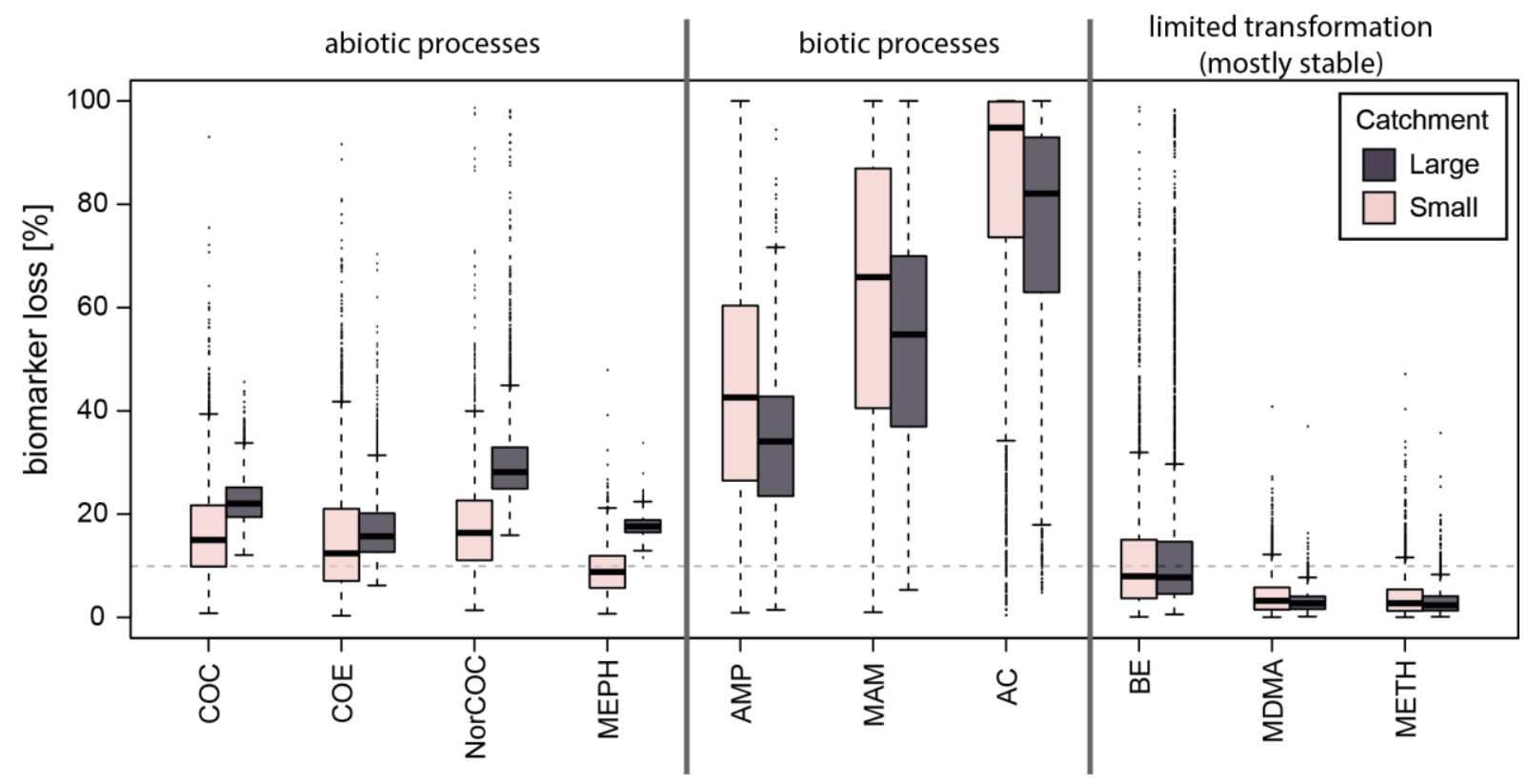

Figure 6. In-sewer transformation for selected biomarkers (scenario 2) calculated for daily mean flow conditions (neglecting temporal variability and dispersion; approach C) for the large and the small catchments. Dashed horizontal line indicates $10 \%$ loss.
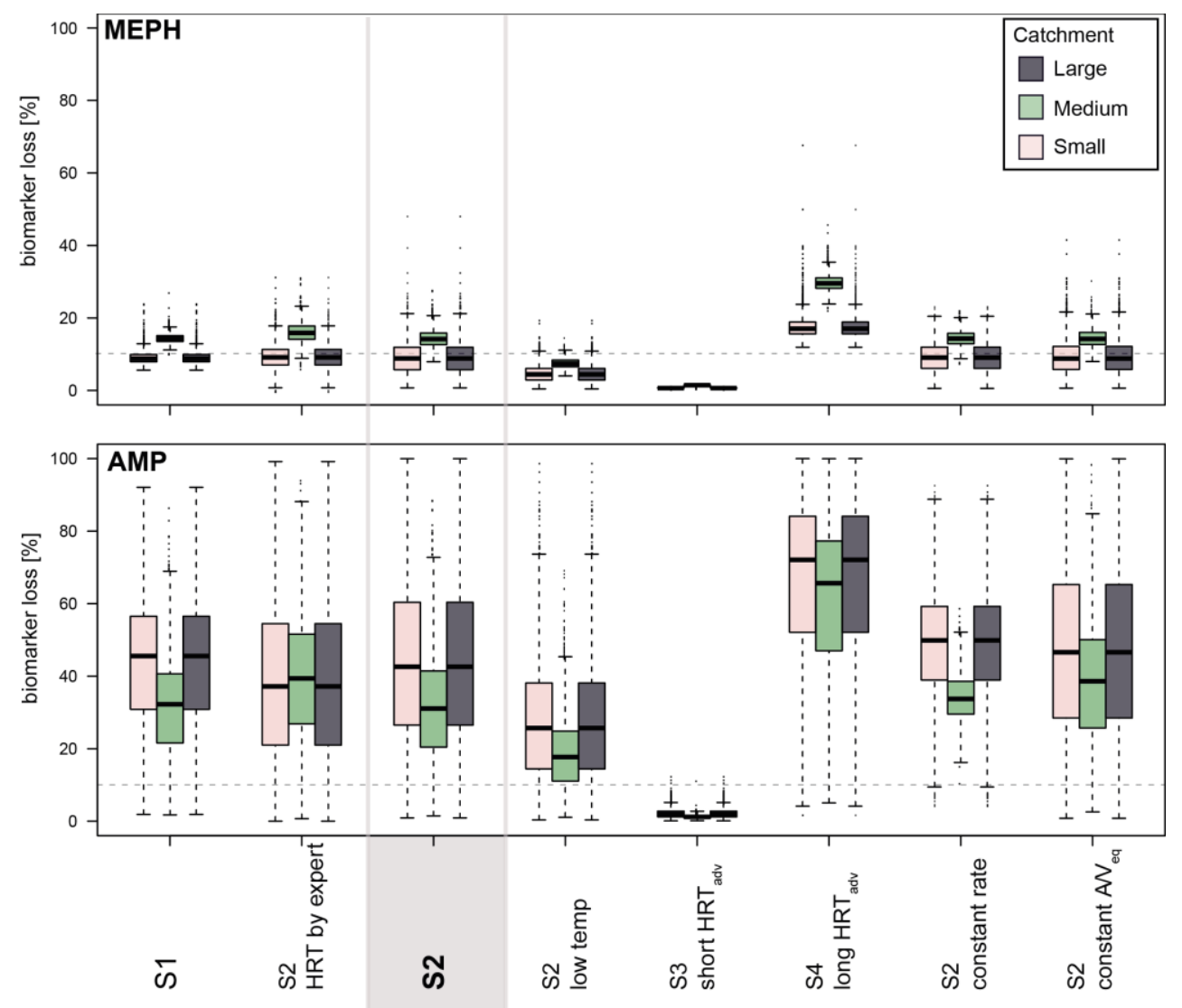

Figure 7. Comparison of biomarker loss for amphetamine (AMP), and mephedrone (MEPH), as boxplots for the small, medium, and large catchments. If not otherwise indicated, results are shown for approach $\mathrm{C}$ for scenarios S1-S4. In the sensitivity analysis the effect of constant, mean rate coefficients (constant rates) and constant $\mathrm{A} / \mathrm{V}_{\text {eq }}$ ratios of $98 \mathrm{~m}^{-1}, 31 \mathrm{~m}^{-1}$, and $30 \mathrm{~m}^{-1}$ (constant $\mathrm{A} / \mathrm{V}$ ) are illustrated for the small, medium and large catchment, respectively. Dashed horizontal line indicates 10\% loss. 


\subsection{Experimental evidence from full-scale study “Glattstollen”}

Temperature and $\mathrm{pH}$ were surprisingly constant over time and similar on both monitoring days over the entire distance (Table 5). Due to a vortex shaft at the beginning of the "Glattstollen", oxygen concentrations are high at L1 and showed high variation at L1 and L3 throughout the 24-h monitoring period (see SI5 for temporal profiles). As expected, oxygen concentrations diminished to a larger extent between L1 and L3 in the presence of biofilm.

Fourteen biomarkers with concentrations $>$ LOQ at both locations (L1 and L3) are plotted in Figure 8.

Nine biomarkers showed transformation of $<10 \%$. The results are compared to model predictions and discussed in section 4.3.4 in more detail.

Table 5. Environmental conditions in the "Glattstollen" during the two sampling campaigns [24-h means and relative standard deviations (RSD)]. Monitoring locations were at L1 (200 m), L2 (2400 m) and L3 (5300 m).

\begin{tabular}{|l|l|l|l|l|}
\hline \multirow{2}{*}{ Location } & Dissolved oxygen & Temperature & $\mathrm{pH}$ \\
\cline { 3 - 5 } & {$\left[\mathrm{mg} \mathrm{L}^{-1}\right](\mathrm{RSD})$} & {$\left[{ }^{\circ} \mathrm{C}\right](\mathrm{RSD})$} & {$[-](\mathrm{RSD})$} \\
\hline \multirow{2}{*}{$\begin{array}{l}\text { with biofilm } \\
08.01 .2015\end{array}$} & $\mathrm{~L} 1$ & $6.9(19 \%)$ & $13.9(5 \%)$ & $8.0(2 \%)$ \\
\cline { 2 - 5 } 11 am for $24 \mathrm{~h}$ & $\mathrm{~L} 2$ & $1.8(72 \%)$ & $14.2(3 \%)$ & $7.7(3 \%)$ \\
\cline { 2 - 5 } without biofilm & L3 & $1.5(75 \%)$ & $14.0(2 \%)$ & $7.6(6 \%)$ \\
19.03 .2015 & L1 & $4.6(39 \%)$ & $15.3(6 \%)$ & $8.0(2 \%)$ \\
\cline { 2 - 5 } 11 am for $23 \mathrm{~h}$ & L2 & $5.3(41 \%)$ & $15.3(4 \%)$ & $7.7(4 \%)$ \\
\cline { 2 - 5 } & L3 & $3.8(47 \%)$ & $14.8(2 \%)$ & $7.8(2 \%)$ \\
\hline
\end{tabular}



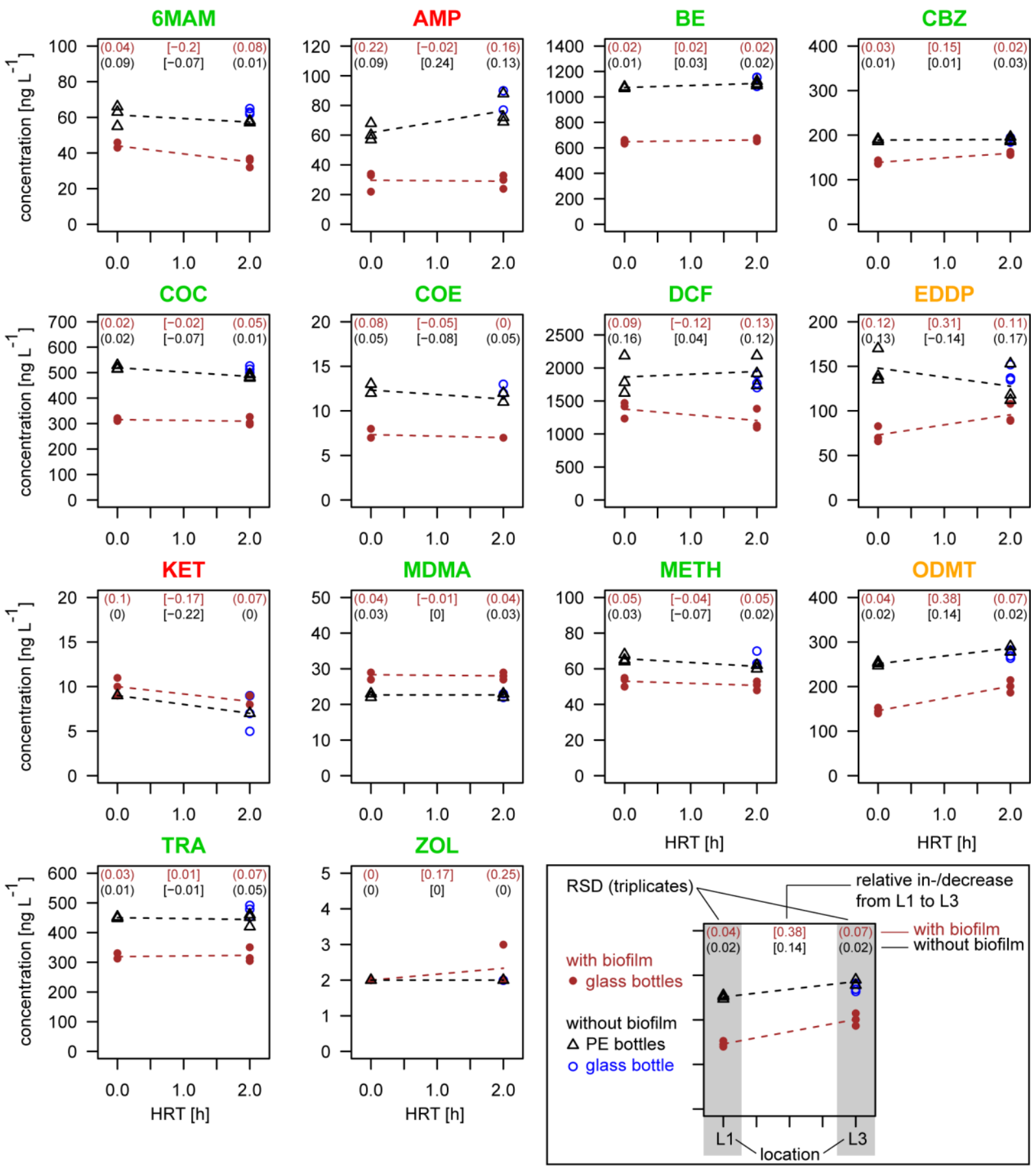

Figure 8. Results from the case study "Glattstollen" (Zürich, Switzerland). Concentrations of biomarkers in 24-h composite samples collected at locations L1 $(200 \mathrm{~m}, \mathrm{t}=0 \mathrm{~h})$ and L3 $(5300 \mathrm{~m}, \mathrm{t}=2 \mathrm{~h})$. See 2.6 for more details. 


\section{Discussion}

Most sewer catchments exhibit high temporal (diurnal) and spatial (population distribution, sewer topology) variability for most hydraulic parameters (e.g., flow rate, fill level, $A / V$ ). It requires increased efforts to explicitly extract and quantify this variability in high resolution. Therefore, extent and magnitude are often unknown. We characterized the relevant hydraulics at catchment-scale by deriving distributions for $H R T$ and $A / V_{e q}$ and separated temporal and spatial variability (4.2). Using these distributions in subsequent water quality modeling, implies low computational cost, when assuming different scenarios for many different biomarkers to quantify potential losses (4.3). To facilitate comparison of simulation results' overall variability, we first discuss variability of transformation rates alone (4.1).

\subsection{Variability in transformation rates}

The variability in biomarker loss can be as high as $40 \%$, considering the performance of only one biofilm (McCall et al., 2016b). When inferring rate coefficients based on four different gravity sewer biofilms (McCall et al., 2016b), the variability of biomarker transformation rate coefficients increases to $80 \%$. The predicative uncertainty became larger due to the environmental variability of the different biofilms. $A / V$. Biomarkers with overall half-lives below 12 hours were the main target of in-sewer simulations (Table 3).

\subsection{Hydraulic conditions}

We determined catchment-representative HRT frequency distributions for the daily mean hydraulic conditions based on different approaches with varying level of hydraulic complexity (Table 1). Modeling hydraulics with and without considering effects of dispersion resulted in similar HRT 
frequency distributions for the three catchment scales ( $H R T_{a d v}$ approach $C$ vs. $H R T_{\text {disp }}$ approach $\left.B\right)$ (Table 4 and Figure S5 in SI6). With growing catchment size, dispersion becomes more important and the difference between the two approaches increased. However, effect of dispersion on overall biomarker loss is negligible. Consequently, it was sufficient to account for advective mass transport only to characterize relevant, realistic $H R T$ distributions $\left(H R T_{a d v}\right)$. To correctly include biofilminduced biodegradation processes in the transformation model, catchment-specific $A / V_{\text {eq }}$ for each flow path were derived using the same approach. Subsequently, we used $H R T_{a d v}$ (approach $C$ ).

\subsubsection{Temporal variability}

415 During 24 hours, the dry weather flow conditions in all conduits of a catchment can vary, mostly due to diurnal patterns of using domestic household appliances. In upper reaches of the network these water packets (flows) enter peripheral pipes over short durations resulting in non-steady, intermittent flow. In transport/trunk sewers of the lower parts of a catchment, flows can still vary, but are substantially higher of continuous nature (Butler and Graham, 1995). Furthermore, a water packet entering the sewer at $1 \mathrm{am}$, travelling for several hours, experiences different conditions along the flow path than a water packet entering at $8 \mathrm{am}$. It is difficult to separate the effects of these intertwined temporal and spatial variables. Therefore, we used the daily mean of the variable hydraulic conditions ( $A / V$, velocity, fill level) to calculate $H R T_{a d v}$ (and $A / V_{\text {eq }}$ ) from each node to the WWTP. The neglected temporal variability was separately quantified by finding the minimum and maximum velocity and fill level at highest temporal resolution $(20 \mathrm{~s})$ in each conduit. From those extreme flow conditions for all flow paths, $H R T_{\max } H R T_{\min }$ and $A / V_{\text {eq, } \max }, A / V_{\text {eq, } \min }$ were calculated and compared with the mean conditions. Even though, it is unlikely that a water packet would experience all minima or maxima conditions chronologically along the flow path, these results provide an estimate of the maximal range of diurnal variability. The resulting ranges for the large catchment were $+20 \%$ to $-10 \%$ for $H R T$ and 
$+35 \%$ to $-20 \%$ for $A / V_{e q}$ (Figure 2; Figure 3). This was a worst-case scenario and real differences are certainly not deviating more than this from our presented mean.

\subsubsection{Spatial variability}

In all catchments $H R T_{a d v}$ increased with path length and distance from the WWTP (Figure 4C). Similarly, $A / V_{e q}$ increased with path length for the medium and large catchment but the trend was more random and mean values were significantly higher in the small catchment (Figure 4D). The medium and large catchments had similar $A / V_{e q}$ distributions with mean values of $31 \mathrm{~m}^{-1}$ and $30 \mathrm{~m}^{-1}$, respectively. However, the mean $A / V_{e q}$ for the small catchment was $98 \mathrm{~m}^{-1}$ with a much wider range (Table 4).

In the small catchment, mean $A / V_{e q}$ were considerably higher compared to the other catchments, due to several peripheral conduits that had very high $A / V_{i}\left(\right.$ up to $\left.1000 \mathrm{~m}^{-1}\right)$, irrespective of their distance from the WWTP (Figure 4D). Smaller catchments (low mean HRTs - including peripheral upper subcatchments in large networks) can have many conduits with small diameters and lower (often intermittent) diurnal flows and, therefore, fill levels. Since $A / V_{i}$ exponentially increases with decreasing fill level, this may have led to the high $A / V_{e q}$ values for the small catchment. This is because we assumed a continuous wastewater production per PE per day following a bimodal, diurnal profile (with flows at any time $>0$ ) and averaged flow to daily mean conditions. This seems appropriate for conduits with continuous, yet variable, flow. In peripheral conduits with intermittent flows, averaging over a 24-h period leads to unrealistic low mean flows (and consequently unrealistic high $A / V$ ) (see SI8 for further discussion). Further research should i) implement more realistic (intermittent) flow conditions for the upper conduits and, ii) more catchments should be characterized in $A / V_{\text {eq }}$ (and HRT) distributions, to confirm the spatio-temporal trends that we identified in the three catchments investigated. 


\subsection{Biomarker loss in different scenarios}

AMP, MAM and AC were found to be unstable with median losses above $40 \%$ and a high variability ranging from 5\% to $100 \%$ losses (Figure 5; Figure 6). Less than $10 \%$ loss in all catchments occurred for MEPH, MDMA, MPA, PMMA, TRA, ZOL, KET, CBZ, DCF, MDPV, MTO, METH, PMA and EDDP. In most scenarios the median loss of COC, COE, NorCOC, HMMA, BE, NorKET and ODMT was below $30 \%$ with varying ranges of variability.

\subsubsection{Influence of catchment scale, prevalence and location}

The catchment scale influenced biomarker loss. As expected, with increasing catchment size, for biomarkers with high abiotic rate coefficients the loss increased, due to the higher $H R T_{a d v}$. In contrast, for biomarkers with high $k_{\text {biffilm, }}$, the median loss in the small catchment was equally high, and higher compared to the large catchment (Figure 6). This counter-intuitive high loss was caused by the higher mean $A / V_{e q}$ despite a small mean $H R T_{a d v}$ in the small catchment (4.1.2). Furthermore, the median biomarker loss was unaffected by prevalence, however, the predicted range of loss increased with lower numbers of drug users in a catchment (see e.g., AMP in Figure 7, S1 (1\% drug users) vs. S2 (0.01\% drug users).

If the drug users were located close to the WWTP (S3) biomarker loss was negligible in all catchments, while the opposite was true for the scenario assuming all drug users to be located far away from the WWTP (Figure 7 - S4). These extreme scenarios seem very unlikely if such drug user distributions are not supported by detailed epidemiological studies available in the catchment under investigation.

\subsubsection{Influence of variability of $A / V_{e q}$ and transformation rates}

A sensitivity analysis showed that results from simulations with constant mean values for $A / V_{e q}$ in the entire catchment (mean of the $A / V_{\text {eq }}$ distribution) increased biomarker loss (Figure 7) but the predicted variation remained about the same (Q3 minus Q1). This is due to the positively skewed $A / V_{\text {eq }}$ distribution (mean higher than mode). 
In contrast, assuming constant transformation rate coefficients substantially reduced biomarker loss uncertainty [similar to constant $A / V_{\text {eq }}$ medians increased partly but variation was reduced (Q3 minus Q1)]. This indicates that reducing variability of transformation rate coefficients could significantly decrease uncertainty of biomarker loss estimates. However, this remains a challenge since the spatiotemporal dynamic nature of prevailing conditions in sewers (environmental and hydraulic) is often unknown, making the implementation of space-dependent transformation rate coefficients based on sewer conditions speculative (see 4.3.3).

Using HRT based on expert guesses (see section 2.5) resulted in estimates comparable with results based on hydrodynamic simulations using "the true" $H R T_{a d v}$ distribution of the catchments. This is obviously only appropriate, if the estimate is accurate. Using the HRT from the local sewer network operator resulted in an overestimation of median biomarker loss in the large catchment and using our $H R T$ estimates for the small, and medium networks resulted in an underestimation of loss (Figure 7).

\subsubsection{Influence of environmental conditions}

Biomarker loss depends on i) environmental conditions and ii) type and performance of biofilm in sewer networks (Hvitved-Jacobsen et al., 2013; McCall et al., 2016b; Thai et al., 2014).

Prevailing environmental factors within a sewer network - e.g. temperature, $\mathrm{pH}$, redox conditions, wastewater composition (e.g., toxic chemicals), accumulation of sediments, etc. - influence transformation rates and vary over space at different time scales (minutes to months).

Spatial differences of environmental factors likely imply also spatial differences of biofilm composition, thickness - e.g. due to varying shear stress, sloughing due to previous rain events - and performance. Previously, we observed that individual biofilms from peripheral, upper conduits expressed lower transformation potential compared to biofilms from trunk sewers further downstream (McCall et al., 2016b). However, more studies would be needed to generalize this observation. Therefore, and in the 
absence of knowledge on the spatio-temporal distribution of environmental factors, the biofilm performance $\left(k_{\text {biofilm }}\right)$ was assumed to be equal across the entire catchment in each of the 3000 simulation runs. To cover the currently known range of biofilm performance, $k_{b i f f i m}$ was sampled for each run from the predicted performance distribution (see section 2.4).

It remains a challenge to overcome efforts and uncertainties related to systematically assess the spatial distribution of prevailing sewer conditions for entire catchments. Possible solutions to consider transformation rates in an informed, space-dependent manner encompass: i) coupling sewer process modeling concepts (e.g. WATS as in Vollertsen et al., 2015) with our approach, or ii) measuring relevant wastewater parameters in sewers at sufficiently high spatial resolution. The latter is currently not possible at reasonable expenditure and requires developing new surveying technologies. Until successful implementation, our proposed sewer modeling framework including environmental variability in the form of aggregated transformation rates, provides an appropriate approach to efficiently estimate realistic biomarker losses including objective ranges of uncertainty.

\subsubsection{Full-scale study "Glattstollen"}

Transformation of the following nine biomarkers was $<10 \%$ (or smaller than rsd from triplicate analyses at L1 or L3) over the two-hour HRT for both experiments, with and without biofilm: BE, CBZ (increase in the presence of biofilm likely due to back-transformation of a CBZ metabolite), COC, COE, DCF, MDMA, METH, TRA, and ZOL. This was expected from model results for the "Glattstollen" (applying eq. 3, HRT $=2 \mathrm{~h}, \mathrm{~A} / \mathrm{V}=11 \mathrm{~m}^{-1}$, rates from SI4, Table S3). For MAM a loss of $20 \%$ was observed in the presence of biofilm, which is within the expected range of model predictions

520 (median loss of $29 \%, \mathrm{q} 10=6 \%, \mathrm{q} 90=48 \%$ ). In the experiment without biofilm a loss of only $7 \%$ was observed (model prediction with no biofilm activity: median loss $4 \%$, $\mathrm{q} 10=2 \%$, $\mathrm{q} 90=6 \%$ ). For EDDP itself no transformation would have been expected with the model, but experimentally we found an increase in the presence of biofilm. In the absence of quantifiable methadone concentrations, it 
remains speculative if this is due to transformation of another methadone metabolite. Similarly, an increase of ODMT was observed in the experiment, while TRA concentrations (parent of ODMT) were similar in L1 and L3 samples. The model predicted a decrease for AMP concentrations in both experiments, while it was stable in the presence of biofilm and even increased in the absence of biofilm in our full-scale experiment. It seems unlikely that AMP was formed as a metabolite of METH, since METH concentrations remained constant. For KET no transformation would have been expected from model results over the $2 \mathrm{~h}$ period. For AMP and KET we currently have no explanation why they deviated from model predictions, warranting further research.

\section{Conclusion}

The study's objectives were to predict biomarker loss during transport in sewers, to estimate related uncertainty and to perform a transformation experiment in a real sewer. We developed a simulation approach that accounts for catchment and flow-path specific hydraulic properties influencing biomarker transformation processes $\left(H R T\right.$ and $\left.A / V_{\text {eq }}\right)$. With this approach we were able to separate computationally demanding hydraulic modeling and biomarker transformation calculations. This facilitates efficient testing of different scenarios (prevalence, environmental conditions) by sampling from transformation rate coefficients, $H R T$ and $A / V_{e q}$ distributions to accurately estimate in-sewer losses and uncertainty for various biomarkers and different catchment scale. The obtained results led to the following main conclusions:

\section{Urban water management}

- The biofilm surface area to wastewater volume ratio $(A / V)$ that can vary from conduit to conduit was summarized in an $\mathrm{A} / \mathrm{V}$ equivalent $\left(A / V_{e q}\right)$ for individual flow paths from nodes to the wastewater treatment plant (normalized with the travel time in each conduit along the corresponding flow path). This allowed assigning a representative, realistic value to each node for 
both $A / V_{\text {eq }}$ value and hydraulic residence time $(H R T)$ of the corresponding flow path for subsequent quality simulations.

- Representative $H R T$ and $A / V_{e q}$ distributions can be generated from hydraulic simulations considering advection only. This approach allowed an adequate quantification of spatial and temporal variability. In a large catchment the temporal variability of $H R T$ and $A / V_{e q}$ differed from the daily mean by $+20 \%$ to $-10 \%$ and $+35 \%$ to $-20 \%$, respectively.

- The resulting spatial distributions, summarizing $H R T s$ and $A / V_{e q}$ for individual flow paths, show a higher mean $H R T$ for the large catchment compared to the small catchment, while for the mean

$555 A / V_{e q}$ it was the opposite with much higher $A / V_{e q}$ for the small than for the large catchment. Overall, effects of unknown spatial variability, were much higher (0-100\% predicted loss) than effects of temporal variability.

- Topography and network topology affect $H R T$ and $A / V_{e q}$ distributions and environmental conditions in sewers. This should be assessed for other locations in future studies.

- Transformation experiments in real sewers are laborious to perform. While observed losses are valuable as plausibility checks, transferability of results to predict transformation rates and range of loss in other catchments is limited.

\section{Wastewater-based epidemiology}

- Median biomarker losses were equally affected by $A / V_{\text {eq }}$ and $H R T$ for the small catchment (small mean $H R T$ and high mean $A / V_{e q}$ ) and large catchment (high mean $H R T$ and small mean $A / V_{e q}$ ). Losses for biomarkers with high abiotic rate coefficients had higher losses in the large catchment. In this study, high rate coefficients $\left(k_{\text {biffilm }}>0.005 \mathrm{~m} \mathrm{~d}^{-1}\right)$ of biofilm resulted in higher median losses of amphetamine, monoacetylmorphine, and acetylcodeine, surprisingly, for the small catchment. 
However, for many biomarkers (methamphetamine, ketamine, MDPV, MDMA, benzoylecgonine) in-sewer losses were negligible in e.g., the back-calculation of illicit drug use.

- Simulation results showed that the uncertainty due variability in transformation rate coefficients is typically higher (or about equal, depending on catchment characteristics) than uncertainty due to unknown drug user location. The latter cannot be reduced easily, which implies that future work should focus on i) improving the knowledge about variability of biomarker transformation rates under different in-sewer conditions and ii) developing a method to efficiently quantify these conditions in real networks at high spatio-temporal resolution.

\section{Acknowledgements}

The authors thank Claudia Bänninger-Werffeli, Philipp Beutler, Tobias Doppler, Jack Eugster, Karin Hauser, Hannes Jenni, Lena Mutzner, Jörg Rieckermann, Karin Rottermann, Andreas Scheidegger, Brian Sinnet, Basilius Thalmann, Jacqueline Traber, and Omar Wani for their support during experimental work and/or valuable input related to methods and writing. We are very grateful for access to "Glattstollen" and support during experiments: Peter Liechti, Roger Küenzi, Beat Rohrbach, Hugo Koller and Hans Lamp (ERZ, Urban Drainage Departement, Zurich, Switzerland). Financial support by the European Union's Seventh Framework Program for research, technological development and demonstration SEWPROF (project no. 317205) is gratefully acknowledged for the experimental part of this study. Furthermore, we thank Stadtentwässerung Dresden for providing details on the sewer network including measured flow data and acknowledge the work of several research assistants at TU Dresden who contributed to the development of the SWMM model. In particular, we thank MSc. Bibek Karanjit for his contribution to the model validation for dry weather conditions. 


\section{References}

Butler, D., Graham, N.J.D., 1995. Modeling Dry Weather Waste-Water Flow in Sewer Networks. J. Environ. Eng. 121, 161-173. doi:10.1061/(ASCE)0733-9372(1995)121:2(161)

Carballa, M., Omil, F., Lema, J.M., 2008. Comparison of predicted and measured concentrations of selected pharmaceuticals, fragrances and hormones in Spanish sewage. Chemosphere 72, 1118 1123. doi:10.1016/j.chemosphere.2008.04.034

Csardi, G., Nepusz, T., 2006. The igraph software package for complex network research. InterJournal Complex Sy, 1695.

Daouk, S., Chèvre, N., Vernaz, N., Widmer, C., Daali, Y., Fleury-Souverain, S., 2016. Dynamics of active pharmaceutical ingredients loads in a Swiss university hospital wastewaters and prediction of the related environmental risk for the aquatic ecosystems. Sci. Total Environ. 547, 244-253. doi:10.1016/j.scitotenv.2015.12.117

Heberer, T., Feldmann, D., 2005. Contribution of effluents from hospitals and private households to the total loads of diclofenac and carbamazepine in municipal sewage effluents - Modeling versus measurements. J. Hazard. Mater. 122, 211-218. doi:10.1016/j.jhazmat.2005.03.007

Hvitved-Jacobsen, T., Vollertsen, J., Nielsen, A.H., 2013. Sewer Processes: Microbial and Chemical Process Engineering of Sewer Networks, Second Edition, second. ed. CRC Press, Boca Raton, FL.

Jelic, A., Rodriguez-Mozaz, S., Barceló, D., Gutierrez, O., 2015. Impact of in-sewer transformation on 43 pharmaceuticals in a pressurized sewer under anaerobic conditions. Water Res. 68, 98-108. doi:10.1016/j.watres.2014.09.033

Kaegi, R., Voegelin, A., Ort, C., Sinnet, B., Thalmann, B., Krismer, J., Hagendorfer, H., Elumelu, M., 
Mueller, E., 2013. Fate and transformation of silver nanoparticles in urban wastewater systems. Water Res. 47, 3866-3877. doi:10.1016/j.watres.2012.11.060

Karpf, C., Krebs, P., 2011. Quantification of groundwater infiltration and surface water inflows in urban sewer networks based on a multiple model approach. Water Res. 45(10), 3129-3136. doi:10.1016/j.watres.2011.03.022

Mathieu, C., Rieckermann, J., Berset, J.D., Sch??rch, S., Brenneisen, R., 2011. Assessment of total uncertainty in cocaine and benzoylecgonine wastewater load measurements. Water Res. 45, 6650-6660. doi:10.1016/j.watres.2011.09.049

McCall, A.-K., Bade, R., Kinyua, J., Lai, F.Y., Thai, P.K., Covaci, A., Bijlsma, L., van Nuijs, A.L.N., Ort, C., 2016a. Critical review on the stability of illicit drugs in sewers and wastewater samples. Water Res. 88, 933-947. doi:10.1016/j.watres.2015.10.040

McCall, A.-K., Scheidegger, A., Madry, M.M., Steuer, A.E., Weissbrodt, D.G., Vanrolleghem, P.A., Kraemer, T., Morgenroth, E., Ort, C., 2016b. Influence of Different Sewer Biofilms on Transformation Rates of Drugs. Environ. Sci. Technol. 50, 13351-13360. doi:10.1021/acs.est.6b04200

Nielsen, P.H., Raunkjaer, K., Norsker, N.H., Jensen, N.A., Hvitved-Jacobsen, T., 1992. Transformation of wastewater in sewer systems - A review, in: Water Science and Technology. Copenhagen, Den, pp. 17-31. Fatta-Kassinos, D., Griffiths, P., Hernández, F., González-Mariño, I., Grabic, R., KasprzykHordern, B., Mastroianni, N., Meierjohann, A., Nefau, T., Östman, M., Pico, Y., Racamonde, I., Reid, M., Slobodnik, J., Terzic, S., Thomaidis, N., Thomas, K. V, 2014. Spatial differences and 
temporal changes in illicit drug use in Europe quantified by wastewater analysis. Addiction 109,

R-Core-Team, 2015. R: A language and environment for statistical computing.

Rossman L.A., 2010. Storm Water Management Model User's Manual Version 5.0, EPA/600/R-05/040, US EPA. National Risk Management Research Laboratory, Cincinnati, Ohio, USA. https://www.epa.gov/water-research/storm-water-management-model-swmm

Ramin, P., Libonati Brock, A., Polesel, F., Causanilles, A., Emke, E., de Voogt, P., Plósz, B.G., 2016. Transformation and Sorption of Illicit Drug Biomarkers in Sewer Systems: Understanding the Role of Suspended Solids in Raw Wastewater. Environ. Sci. Technol. 50, 13397-13408. doi:10.1021/acs.est.6b03049

Suárez, S., Reif, R., Lema, J.M., Omil, F., 2012. Mass balance of pharmaceutical and personal care products in a pilot-scale single-sludge system: Influence of T, SRT and recirculation ratio. Chemosphere 89, 164-171. doi:10.1016/j.chemosphere.2012.05.094

Thai, P.K., Jiang, G., Gernjak, W., Yuan, Z., Lai, F.Y., Mueller, J.F., 2014. Effects of sewer conditions on the degradation of selected illicit drug residues in wastewater. Water Res. 48, 538-547. doi:10.1016/j.watres.2013.10.019 F., Vanrolleghem, P. a., Mikkelsen, P.S., 2014. A model library for dynamic transport and fate of micropollutants in integrated urban wastewater and stormwater systems. Environ. Model. Softw. 53, 98-111. doi:10.1016/j.envsoft.2013.11.010

Vollertsen, J., Revilla, N., Hvitved-Jacobsen, T., Nielsen, A.H., 2015. Modeling sulfides, pH and hydrogen sulfide gas in the sewers of San Francisco. Water Environment Research 87(11), 1980- 
1989. doi:10.2175/106143015X14362865226752 